Sādhanā Vol. 29, Part 4, August 2004, pp. 389-400. @ Printed in India

\title{
Application of holography in jet acoustic studies
}

\author{
G PUNDARIKA $^{1}$, R LAKSHMINARAYANA $^{2}$ and T S SHESHADRI ${ }^{2}$ \\ ${ }^{1}$ Department of Mechanical Engineering, BMS College of Engineering, \\ Bangalore 560 019, India \\ ${ }^{2}$ Department of Aerospace Engineering, Indian Institute of Science, \\ Bangalore 560 012, India \\ e-mail: ${ }^{1}$ gpkbms@yahoo.com; ${ }^{2}\{$ apdl,tss $\} @$ aero.iisc.ernet.in
}

MS received 4 February 2003; revised 30 April 2004

\begin{abstract}
Source strength distribution on a jet boundary was obtained from measurements using the principle of acoustic holography. Measurements were conducted in an open field. Measurement of acoustic pressure on a cylindrical twodimensional contour located close to the vibrating jet boundary was used to obtain the acoustic source strength distribution at the jet boundary. Particular attention was focussed on back projection of the sound field on to a cylindrical surface. A jet emanating from $5 \mathrm{~mm}$ convergent nozzle was used for the holography experiments, assuming axisymmetry. Experimental results were compared with results obtained from holography.
\end{abstract}

Keywords. Acoustic noise; jet noise; source strength distribution.

\section{Introduction}

Holography is a technique by which acoustic imaging is done. It arises from the high information content; that is, data recorded on a two-dimensional surface (the hologram) may be used to reconstruct an entire three-dimensional wave field, with the well-known result of obtaining three-dimensional images. It has utility in many different fields, particularly in the application of acoustic holography to study the noise radiation in the audio frequency region.

One must be able to measure the sound field in the near field of the radiator, generally at a measurement distance of less than several centimetres. This closeness is necessary to capture the exponentially decaying, evanescent wave fields emanating from the source. These waves contain all the high resolution information about the source. This discovery led to the birth of near-field acoustical holography (Williams \& Maynard 1980).

Generalized holography involves the measurement of the wave field on an appropriate surface and the use of this measurement to uniquely determine the wave field within a threedimensional region. This description indicates that generalized holography is equivalent to the use of a Dirichlet boundary condition (Morse \& Feshbach 1953) on a surface for which

A list of symbols is given at the end of the paper 
Green's function is known. One usually imagines boundary value problems as having boundary conditions determined by a source (for example, a vibrating surface in an acoustics problem); such problems are difficult because the source may provide conditions for which there is no known Green's function. In generalized holography, one simply measures a uniform (Dirichlet or Neuman) boundary condition on a surface for which there is a known Green's function. The holographic reconstruction process is then simply the convolution (or deconvolution) of the measured boundary values with Green's function. In theory this is a straightforward process; in practice some care must be taken in order to identify and avoid the limitations of conventional holography. The causes of the limitations occur in the method of measuring the boundary data, in the formulation of Green's function, and in the evaluation of the convolution integral.

The basic assumption is that some sources creat a wave field, $\psi(r, t)$, (a function of position $r$ in a three-dimensional region of space and time $t$ ) which, within a three-dimensional region of interest, satisfies the homogeneous wave equation:

$$
\nabla^{2} \psi-\frac{1}{c^{2}} \frac{\partial^{2} \psi}{\partial t^{2}}=0
$$

Here, $\nabla^{2}$ is the Laplacian operator and $c$ is a constant propagation speed. The following is further assumed.

(1) There is a surface $S$ enclosing the three-dimensional region of interest for which there is a known Green's function, $G\left(r \mid r_{s}\right)$, satisfying the homogeneous Helmholtz equation for $r$ inside $S$ and vanishing (or having a vanishing normal derivative) for $r=r_{s}$ on $S$. Part of $S$ may be at infinity; in practice the part of $S$ not at infinity will be a level surface of some separable coordinate system which is in close contact with the sources.

(2) There is a surface $H$ (the hologram surface) which may coincide with $S$ or have a level surface parallel to $S$ for which $\psi\left(r_{H}, t\right)$ (or its normal derivative can be measured or assumed for all $r_{H}$ on $H$ and all $t$.

If the above conditions are met, then $\psi\left(r_{H}, t\right)$ for $r$ inside $S$ can be uniquely determined from $\psi\left(r_{H}, t\right)$ with $r_{H}$ on $H$.

The first step in finding $\psi(r, t)$ from $\psi\left(r_{H}, t\right)$ is to Fourier transform in time:

$$
\tilde{\psi}(r, \omega)=\int_{-\infty}^{\infty} \psi(r, t) e^{i \omega t} \mathrm{~d} t,
$$

and

$$
\tilde{\psi}\left(r_{H}, \omega\right)=\int_{-\infty}^{\infty} \psi\left(r_{H}, t\right) e^{i \omega t} \mathrm{~d} t .
$$

The symbol " " indicates a complex field having an amplitude and phase depending on $r$. The wave equation becomes the Helmholtz equation,

$$
\nabla^{2} \tilde{\psi}(r, \omega)+k^{2} \tilde{\psi}(r, \omega)=0,
$$

with wave number $k=\omega / c$. It should be noted that formally the boundary data $\psi\left(r_{S}, t\right)$ must be measured for all time $-\infty \leqslant t \leqslant \infty$. Here, $\psi\left(r_{S}, t\right)$ may be measured within a finite 
time window of duration $T$ if $\psi\left(r_{S}, t\right)$ is known to be periodic with period $T$. For a noise source, one may assume that there exists a time scale $T$ for which statistical averages become stationary within specified limits of fluctuation (Kreyszig 1993); in this case also a finite Fourier transform is sufficient. For most noise sources, a reasonable $T$ can be used; however, there are exceptions where $T$ may be so large as to preclude the acquisition of manageable amount of data. An example would be a high-frequency transient in a highly reverberant room. In digital holography, $\psi\left(r_{H}, t\right)$ is sampled at $N$ discrete points in time $t_{n}=t_{r_{H}}+n T / N$ (noting that the starting time $t_{r_{H}}$ may be different for different positions on the surface $H$ ). It is assumed that the sampling is accomplished at the Nyquist rate to prevent aliasing in the time domain. Expression (3) becomes

$$
\tilde{\psi}\left(r_{H}, \omega_{m}\right) \approx e^{i \omega_{m} t_{r_{H}}}\left(\sum_{n=0}^{N-1} \psi\left(r_{H}, t_{n}\right) e^{i 2 \pi n m / N}\right) \frac{T}{N},
$$

where $\omega_{m}=2 \pi m / T$ and $m$ is a non-negative integer less than $N / 2$. The summation in large parentheses can now be accomplished with a fast Fourier transform (FFT) computer algorithm. The errors associated with (5) are not unique to holography but are common to all signal processing involving discrete, finite window sampling. Discussions of these errors can be found in any text on signal processing (Maynard et al 1985). The more interesting aspects of generalized holography are found in the spatial, rather than the temporal, signal processing. For the purpose of the spatial analysis in the next sections, it can be assumed that the sources are driven at frequencies $\omega_{m}=2 \pi m 2 \pi m / T$ with $m(<N / 2)$ some integer and $T$ and $N$ fixed. For most sources it can be assumed that the wave field generated by these harmonic sources does not differ significantly from the actual wave field. If the actual operating frequency of the source is known, then signal-processing techniques can be used to correct $\tilde{\psi}\left(r_{H}, \omega_{m}\right)$. At any rate, for sources operating at the set of frequencies $\omega_{m}$, expression (5) becomes exact.

For the spatial analysis we consider a fixed value of $\omega$ so that there is a fixed wave number $k=\omega / c$ and a single characteristic wavelength $\lambda=2 \pi c / \omega$. The spatial problem is now to find the complex field $\tilde{\psi}(r)$ satisfying the homogeneous Helmholtz equation,

$$
\nabla^{2} \tilde{\psi}(r)+k^{2} \tilde{\psi}(r)=0
$$

for $r$ within the three-dimensional region of interest, given $\tilde{\psi}\left(r_{H}\right)$ for $r_{H}$ on the hologram surface $H$.

\subsection{Implementation}

Implementation of generalized holography in an actual system involves acquisition of the hologram data and evaluation of the various expressions of generalized holography. Because the features of the (hardware) system used for actual data acquisition depend on many extraneous design variables, few general comments may be made about data acquisition. On the other hand, a number of interesting general comments can be made concerning the numerical evaluation of the holography expressions. The following paragraphs discuss the general features, problems, limitations etc. associated with the actual implementation of generalized holography. In these paragraphs it should be assumed that the comments are about plane holography in particular but may be generalized to other coordinate systems unless stated.

Concerning data acquisition, it can be assumed that the major temporal frequency components are sampled at the Nyquist rate (a theorem, developed by H Nyquist, which states that 
an analogue signal waveform may be uniquely reconstructed, without error, from samples taken at equal time intervals. The sampling rate must be equal to, or greater than, twice the highest frequency component in the analog signal) or faster, and that any other components at higher frequencies are filtered to a sufficiently small "noise" level. The time-sampled data may then be analysed to produce the temporal frequency complex amplitudes $\tilde{\psi}\left(r_{H}\right)$, as discussed. In theory, the hologram data must be known as a continuous function (i.e., known at all points $r_{H}$ ) over the hologram surface $H$ which may be infinite in extent. In practice, the hologram data can only be sampled at discrete points on a surface of finite extent (referred to as the hologram aperture). As far as the discrete sampling is concerned, one must be certain that the field $\tilde{\psi}\left(r_{H}\right)$ is being sampled at the spatial Nyquist rate. It should be recalled that any spatial frequencies of the source, which exceed those of the characteristic radiated wavelengths, exponentially decay with distance from the source. Thus spatial sampling is provided with a natural filter; as an empirical rule-of-thumb, we find that if the hologram sampling is done at a distance $d$ from the source, then the distance between sampling points should be no larger than $d$. Discrete spatial sampling does not result in any unusual problems in generalized holography. On the other hand, the finite hologram aperture does result in fundamental problems, which require special processing techniques. Of course, the holography expressions which involve integrals over infinite domains in space (as in plane and cylindrical holography) necessitate that some assumption be made about the hologram (or source) data, which lie outside the finite hologram aperture. Practical limitations notwithstanding, it can be assumed that the hologram aperture may be made sufficiently larger than the sources (of finite extent) so that the field on the surface beyond the aperture is not significantly different from zero. This is a reasonable assumption for laboratory studies.

\subsection{Formulation of the problem}

The work discussed deals only with planar radiators and becomes impractical for sources that differ severely from a planar shape. Only one reference (Maynard et al 1985) has discussed the theory of forward and backward projection of sound fields on cylindrical surfaces. Not much information is available either on the net or in recent publications to make an in depth analysis. The present work enables the identification of sources, which are cylindrical or nearly cylindrical in shape. Reconstruction of sound fields from the sources is not attempted here. As discussed in numerous texts, and in the review of $\mathrm{NAH}$, radiation problem involved finding a solution $P$ (a complex field) for the Helmholtz equation (Pierce 1981; Kinsler \& Frey 1985),

$$
\nabla^{2} P(r, \theta, z)+k^{2} P(r, \theta, z)=0,
$$

where $k=w / c$. Near-field acoustical holography was performed by measuring the sound pressure field over a defined cylindrical contour (assuming jet boundary is locally cylindrical in shape). The diameter of the jet at $40 d$ downstream from the nozzle exit was estimated using the following experimentally found empirical relations.

(i) Outer boundary of the jet axis $\left(r_{0}\right)$ is given by (Tennekes \& Lumley 1997) $r_{o}=(d / 2)+$ $0 \cdot 137 z$, where $d$ is the exit diameter of the nozzle and $z$ is the axial distance from the nozzle exit. Taking $d=5 \mathrm{~mm}$ (convergent nozzle of $5 \mathrm{~mm}$ diameter was used for this experiment) and

$$
\begin{aligned}
z & =40 d=40 \times 5=200 \mathrm{~mm}, \\
r_{0} & =(5 / 2)+0 \cdot 137 \times 200, \\
r_{0} & =2 \cdot 5+27.4=29.9 \approx 30 \mathrm{~mm} .
\end{aligned}
$$


This can also be determined by the divergence angle of the jet given experimentally for underexpanded jets (Coleman and Snedeker 1971). The divergence angle lies between $6^{\circ}$ to $8^{\circ}$. Taking $8^{\circ}$,

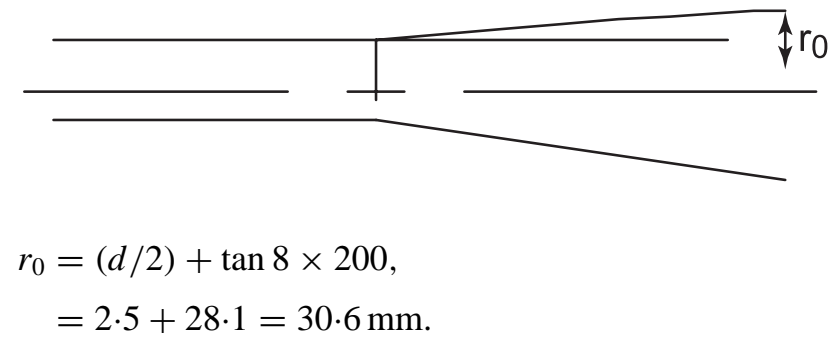

The above results are also consistent with the recent technique used for jet mapping (PIV).

After locating the jet boundary, two cylindrical contours away from the jet boundary at a distance of $5 \mathrm{~cm}$ each were chosen. In the present experiment, taking $\Delta z=L / N$ sampling interval for the pressure field on the contour, $N$ the number of intervals in the axial and radial directions (made equal for sake of simplicity) and $L$ the total length of the measurement aperture in the axial direction $(40 d)$. Also it was assumed that there is no variation in the pressure in the azimuthal direction for a given radius. Back projection of sound pressure from $0.13 \mathrm{~m}$ cylindrical contour was done using (7) as explained in $\S 4, k=w / c=2 \pi f / c$. Taking $f=11250 \mathrm{~Hz}$

and $k_{z}$ (axial wave number) $=\pi / \Delta z$ with $\Delta z=5 \mathrm{~cm}$,

$k_{r}=\left(k^{2}-k_{z}^{2}\right)^{1 / 2}$ and $k_{r} r_{1}, k_{r} r_{2}$ can be found out. These values were used in the Hankel function, which is used in (16). The values of the Hankel functions are obtained from the tables of Bessel "s functions. Numerical values of all the quantities described above are given in the input file (appendix A).

Several authors have discussed the theory of forward and back projection of sound fields on plane surfaces. In this investigation particular attention will be focussed on backward projection of the sound field on to a cylindrical surface. Acoustic holography involves back projection onto a cylindrical surface using measured values of acoustic pressure on an outer cylinder. If the inner surface is chosen to be the jet boundary, one obtains the acoustic source strength distribution at the jet boundary. Back projection calculations have been made using the experimental measurements.

\section{Experimental setup}

The experimental setup consists essentially of a nozzle exhausting into the open atmosphere. Compressed air from a compressed air receiver tank of 70 bar $\left(1\right.$ bar $\left.=10^{5} \mathrm{~Pa}\right)$ and $0.5 \mathrm{~m}^{3}$ capacity, approximately at a room temperature of $24^{\circ} \mathrm{C}$, was supplied to the nozzle via a control valve and a settling pressure chamber. This chamber is a cylinder of diameter $0.421 \mathrm{~m}$ and length $0.85 \mathrm{~m}$, and is designed to withstand 49.05 bar pressure.

One convergent nozzle of throat diameter $5 \mathrm{~mm}$ was used in the holography experiment. The nozzle was made of mild steel.

The measuring and recording equipment consists of a $1 / 2$ " Bruel \& Kjaer pre-polarised microphone type 4189, which is used in conjunction with a B \& K pre-amplifier type 2669 


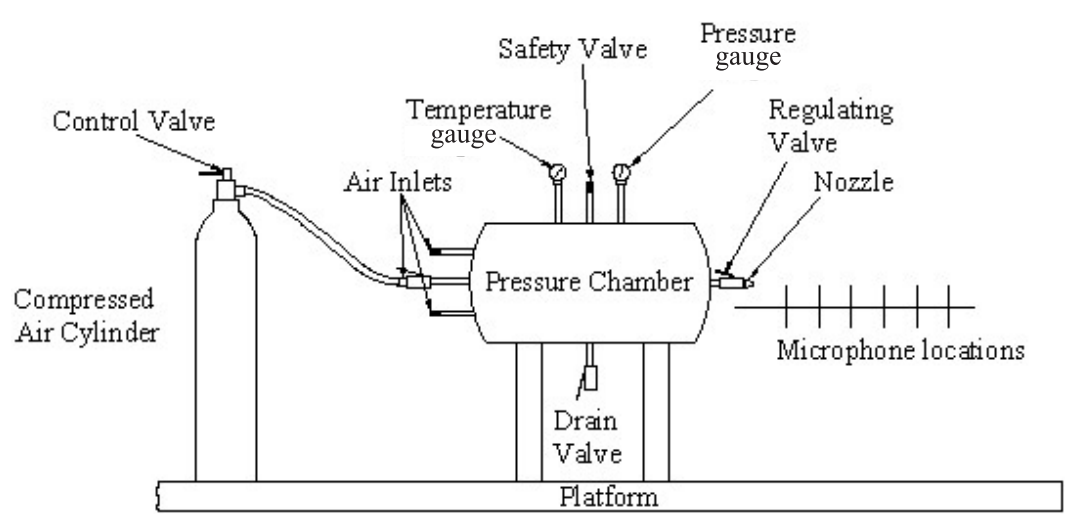

Figure 1. Schematic layout of jet noise rig.

and B \& K conditioning amplifier type 2690. Measurements are made simultaneously with a microphone and a mediator B \& K type 2238 which measures SPL at a point. The microphone can be mounted at various positions of the jet axis using a stand of mild steel framework. Microphone holders are positioned $90^{\circ}$ to the jet axis. Figure 1 shows the schematic layout of an experimental setup.

\section{Measurement details}

Measurements of sound pressure level between the frequencies of $20 \mathrm{~Hz}$ to $20 \mathrm{kHz}$ (audible range) have been made along the jet axis for a choked nozzle having an exit velocity of $316 \mathrm{~m} / \mathrm{s}$. Measurements were recorded upto $40 d$ ( $d$ is the diameter of the nozzle exit) from the nozzle exit and radially $0.08 \mathrm{~m}$ and $0.13 \mathrm{~m}$ at an interval of $10 \mathrm{~d}$ each $(5$ measurement points) with a chamber stagnation pressure of 14.706 bar. Two cylindrical contours each of radius $0.13 \mathrm{~m}$ and $0.08 \mathrm{~m}$ from the jet boundary were used. The jet boundary had a diameter of about $0.06 \mathrm{~m}$ at $40 \mathrm{~d}$. Figure 2 shows measurement points around the jet.

\section{Back projection of acoustic pressure on a cylindrical contour}

Considering a homogeneous wave equation that satisfies the wave field which is created by some noise sources as a function of space and time in a three-dimensional region,

$$
\nabla^{2} p^{\prime}-\frac{1}{c^{2}} \frac{\partial^{2} p^{\prime}}{\partial t^{2}}=0
$$

Here, $\nabla$ is a Laplacian operator and $c$ is a constant propagation speed. Taking $p^{\prime}=R_{e} p e^{-i \omega t}$ and $v^{\prime}=R_{e} v e^{-i \omega t}$, on substitution and simplification (8) reduces to

$$
\nabla^{2} p(r, \theta, z)+k^{2} p(r, \theta, z)=0 .
$$

Equation (9) is a homogeneous Helmholtz equation for the acoustic pressure $p$, where $k=$ $\omega / c, \omega$ is the angular frequency and $c$ is the speed of sound in air, $\theta$ is the circumferential angle and $z$ is the axis of the jet. It is assumed that the source is a finite cylinder of radius $a$ 


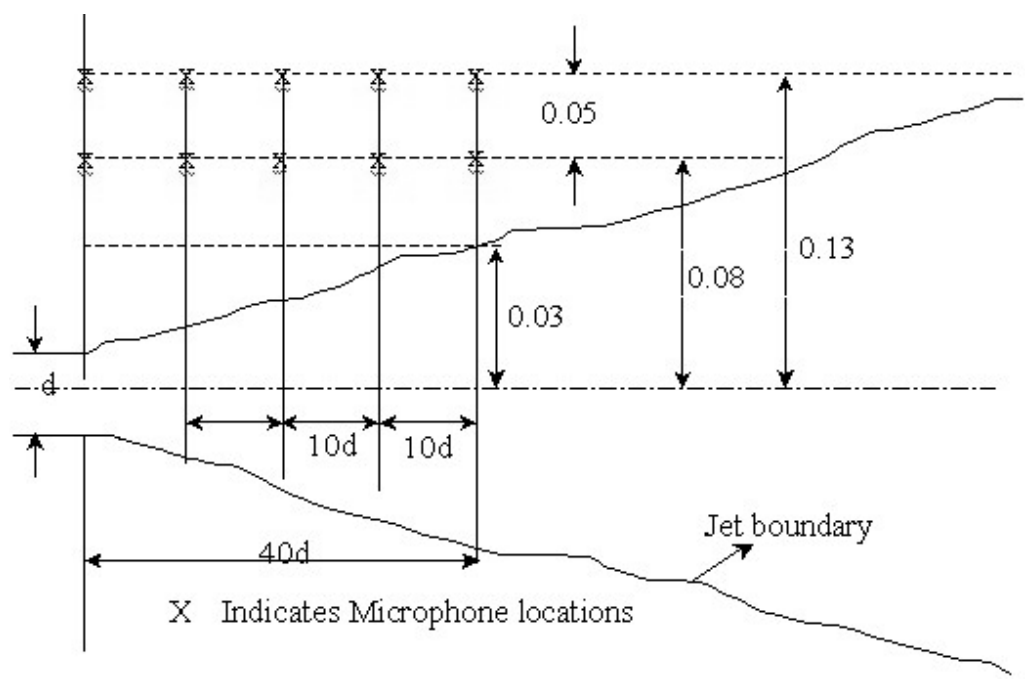

Figure 2. Microphone locations around the jet boundary.

in an otherwise source free region. Equation (9) holds for $r>a$ on any cylindrical surface ( $r=r_{1}=$ constant $)$ outside of this source, the pressure field can be expanded in terms of the cylindrical wave functions $e^{i n \theta}$ and $e^{i k_{z} z}$ as

$$
p\left(r_{1}, \theta, z\right)=\sum_{n=-\infty}^{\infty} \frac{1}{2 \pi} \int_{-\infty}^{\infty} \mathrm{d} k_{z} p_{n}\left(r_{1}, k_{z}\right) e^{i n \theta} e^{i k_{z} z}
$$

In the above equation, $k_{z}\left(k_{z}=2 \pi / \lambda_{z}\right)$ is the axial wave number with units of radians per metre and $n$ is the circumferential wave number (the number of wavelengths, which fit around the circumference of the cylindrical jet). $p_{n}\left(r_{1}, k_{z}\right)$ provides the amplitude and phase for each of the cylindrical wavefunctions.

The values of $p_{n}\left(r_{1}, k_{z}\right)$ are obtained by taking Fourier transforms of both sides of (10), that is

$$
p_{n}\left(r_{1}, k_{z}\right)=\frac{1}{2 \pi} \int_{0}^{2 \pi} \mathrm{d} \theta e^{-i n \theta} \int_{-\infty}^{\infty} \mathrm{d} z e^{-i k_{z} z} p\left(r_{1}, \theta, z\right) .
$$

Equation (10) is an inverse two-dimensional Fourier transforms in $\theta$ and $z$, and (11) follows directly from it. Notice that the inverse transform in $\theta$ is a Fourier series, whereas the transform in $z$ is a continuos Fourier transform. Let an operator $\tau$ stand for two-dimensional forward Fourier transform and $\tau^{-1}$ stand for the inverse transform. Then (10) and (11) can be written as

$$
\begin{gathered}
p\left(r_{1}, \theta, z\right)=\tau^{-1}\left\{p_{n}\left(r_{1}, k_{z}\right)\right\}, \\
p_{n}\left(r_{1}, k_{z}\right)=\tau\left\{p\left(r_{1}, \theta, z\right)\right\} .
\end{gathered}
$$

Equation (11) implies that if the acoustic pressure at a particular frequency is known over an infinite surface $r=r_{1}$, then the co-efficients of $p_{n}$ can be found by direct integration. 
In the experiments, pressure was measured on a cylindrical contour for a discrete set $\left(\theta_{i}, z_{i}\right)$ of coordinates assuming no variation of acoustic pressure in the azimuthal direction for a given cylindrical contour. A set of criteria was used which guarantees that the discretisation (computer programming and implementation) is fine enough to provide an accurate approximation of $p_{n}\left(r_{1}, k_{z}\right)$. Assuming that $p_{n}$ is determined accurately from experimental data. The pressure on a different surface at $r=r_{2}$ can be determined by rewriting (10) for $r=r_{2}$ as

$$
p\left(r_{2}, \theta, z\right)=\sum_{n=-\infty}^{\infty}\left(\frac{1}{2 \pi}\right) \int_{-\infty}^{\infty} \mathrm{d} k_{z} p_{n}\left(r_{2}, k_{z}\right) e^{i n \theta} e^{i k_{z} z},
$$

or equivalently,

$$
p\left(r_{2}, \theta, z\right)=\tau^{-1}\left\{p_{n}\left(r_{2}, k_{z}\right)\right\}
$$

The relations between $p_{n}\left(r_{2}, k_{z}\right)$ and $p_{n}\left(r_{1}, k_{z}\right)$ can be obtained from the radial part of (9), (after separation of variables and Fourier transformation) that is,

$$
p_{n}\left(r_{2}, k_{z}\right)=\left[\frac{H_{v}^{(1)}\left(k_{r}, r_{2}\right)}{H_{v}^{(1)}\left(k_{r}, r_{1}\right)}\right] p_{n}\left(r_{1}, k_{z}\right),
$$

where,

$$
\begin{aligned}
k_{r} & =\left(k^{2}-k_{z}^{2}\right)^{1 / 2}, \\
H_{v}^{(1)} & =J_{v}(x)+i Y_{v}(x)=\text { outgoing Hankel function, } \\
Y_{v} & =\text { Neumann's function. }
\end{aligned}
$$

A very important assumption is made in deriving (16); no incoming waves exist so that the other solutions to the radial equation $H_{v}^{(2)}$ are ignored. This is equivalent to assuming that the experiments are done in an anechoic chamber so that no energy is reflected back to the hologram surface from external walls or other structures. In (16), $r_{2}$ may be less than or greater than $r_{1}$, corresponding to back-projection and forward-projection respectively. Equation (16) is the key to projecting pressure fields from one cylindrical contour to another. The acoustic pressure measured at $r=r_{1}$ is constructed at $r=r_{2}$ by inserting (16) into (14).

\section{Implementation problems}

Problems in implementation are due to the following two reasons.

\subsection{Irregular jet boundary}

In this experimental work, the measurement grid was chosen to be $5 \times 5$ points in the $r$ and $z$ directions, each $0.05 \mathrm{~m}$ apart, for a given chamber stagnation pressure. Measurements were made on a jet boundary assuming it as locally cylindrical in shape. Owing to this it is difficult to get a smooth cylindrical contour nearer to the jet boundary that grows linearly with the jet axis. 


\subsection{Evanescent waves}

Equation (16) is well-known and has been used to study forward-wave propagation. However, for backward propagation, new difficulties arise which are not present in forward propagation. In back projection, one must include evanescent waves generated by the vibrating structure, and they cannot be ignored as they are for forward propagation.

Consider the wavelength in the axial direction, given by $\lambda_{z}=2 \pi / k_{z}$ and the effective wavelength in the circumferential direction as $\lambda_{\theta}=2 \pi r_{1} / n$. When the wavelength in the axial direction is smaller than the acoustic wavelength $\lambda(\lambda=2 \pi / k)$, then one would expect that energy propagation into the medium from the surface $r=r_{1}$ is strongly attenuated. These non-propagating waves are called subsonic or evanescent waves and exhibit an exponential decay away from the surface. That is when $\lambda_{z}<\lambda$, then $k_{z}>k$.

These waves contain the fine detail, high resolution and information about the source. We must be able to measure these components, if we want to reconstruct the field on the surface at low frequencies. Since these waves decay rapidly from the surface, one must measure the fields close to the surface, with a measurement system a few centimetres away from the vibrating surface.

In this experimental work, measurements were made at different points in the measurement field each $0.05 \mathrm{~m}$ apart. With the knowledge of theory of turbulence, the approximate diameter of the jet was calculated $40 d$ from the nozzle exit, considering the same contour all along the entire measurement length as shown in figure 2 .

\section{Results and discussion}

Source strength at the jet boundary can be determined by the principle of near-field acoustic holography. Measurements were recorded choosing a distance of $40 \mathrm{~d}$ at an interval of $10 \mathrm{~d}$ each from the nozzle exit ( $d=$ exit diameter of the nozzle) corresponding to a chamber stagnation pressure of 14.7 bar. In this present investigation, a $5 \mathrm{~mm}$ convergent nozzle was used for holography experiments. The approximate diameter of the jet at $40 \mathrm{~d}$ downstream from the nozzle exit was estimated to be $0.06 \mathrm{~m}$ with the knowledge of theory of turbulence. Choosing two cylindrical contours away from the jet boundary at distances of $0.08 \mathrm{~m}$ and $0.13 \mathrm{~m}$. Back projection of acoustic pressure amplitude from $0.13 \mathrm{~m}$ cylindrical contour to $0.08 \mathrm{~m}$ contour was obtained at the dominating average signal frequency of $11.25 \mathrm{kHz}$. It is evident from the figures 3 and 4 that the experimental values at $0.08 \mathrm{~m}$ cylindrical contour very closely match the results obtained from holography.

Acoustic pressure amplitude values obtained from holography were compared with experimental results and there was an error of less than $11 \%$ in terms of acoustic pressure amplitude and about $2 \%$ in terms of SPL. This validates the procedure. Using acoustic pressure amplitude values from the $0.08 \mathrm{~m}$ cylindrical contour, the source strength distribution at the jet boundary was obtained for the locations mentioned above and corresponding sound pressure levels were in the expected range as shown in figure 5. It is evident from this figure that the acoustic pressure as well as SPL increases from the nozzle exit to the downstream measurement points. Figures 3 and 4 show the same variations. This is because of the following reasons: (i) Noise generated by turbulence mixing starts from $4 d$ to $6 d$ from the nozzle exit to up to about 40d (Stone 1974); (ii) Cylindrical jet contour cannot be maintained as it grows linearly with its axis downstream, and therefore measurement points are nearer to the jet boundary as it proceeds downstream from the nozzle exit; (iii) The strong downstream directivity of the noise at low frequency. 


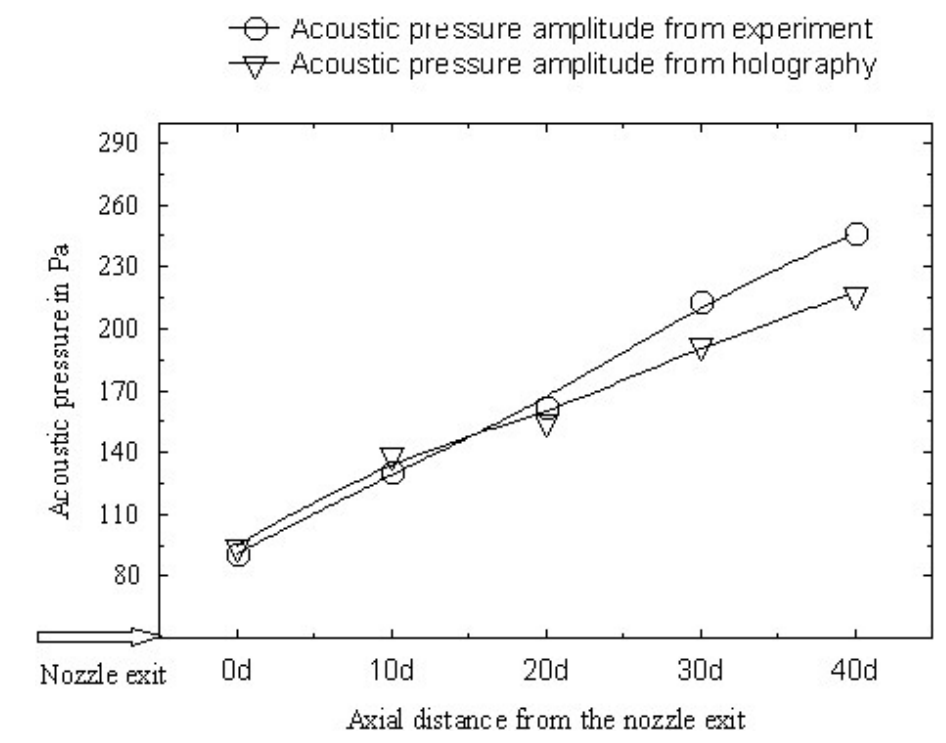

Figure 3. Variation of acoustic pressure amplitude along the jet axis for $0.08 \mathrm{~m}$ cylindrical contours.

\section{Conclusions}

Source strength distribution at the jet boundary has been obtained by the principle of nearfield acoustic holography. This was done by considering the jet boundary as a cylindrical contour and measuring the acoustic pressure amplitude a few centimetres away from the jet boundary, and projecting back to its surface to determine the source strength. The acoustic holography technique adopted here would bring in a new concept of identifying noise source strength from the jets.

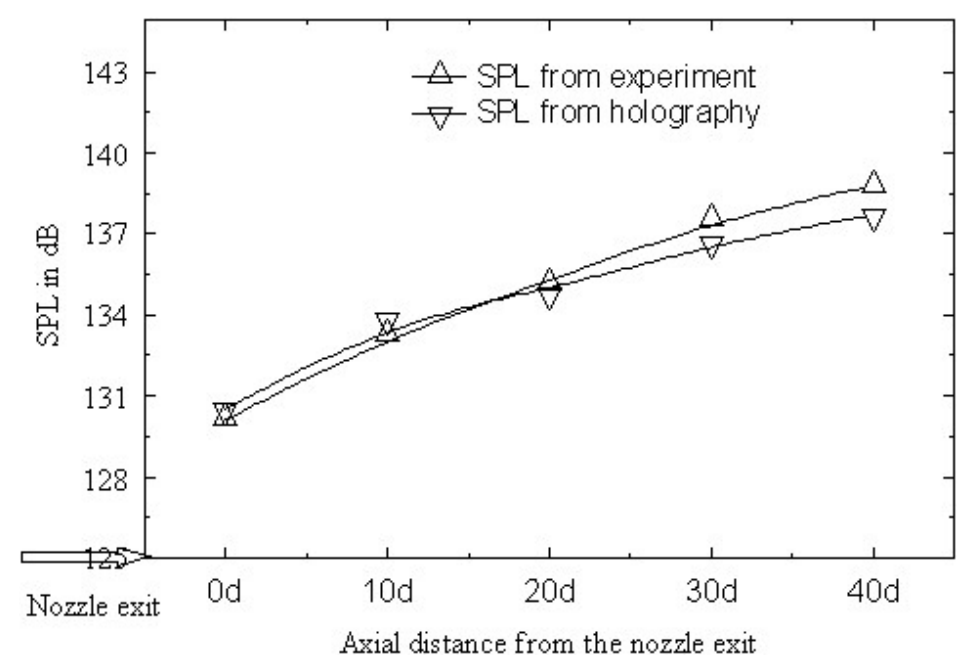

Figure 4. Variation of SPL along the jet axis for $0.08 \mathrm{~m}$ cylindrical contours. 


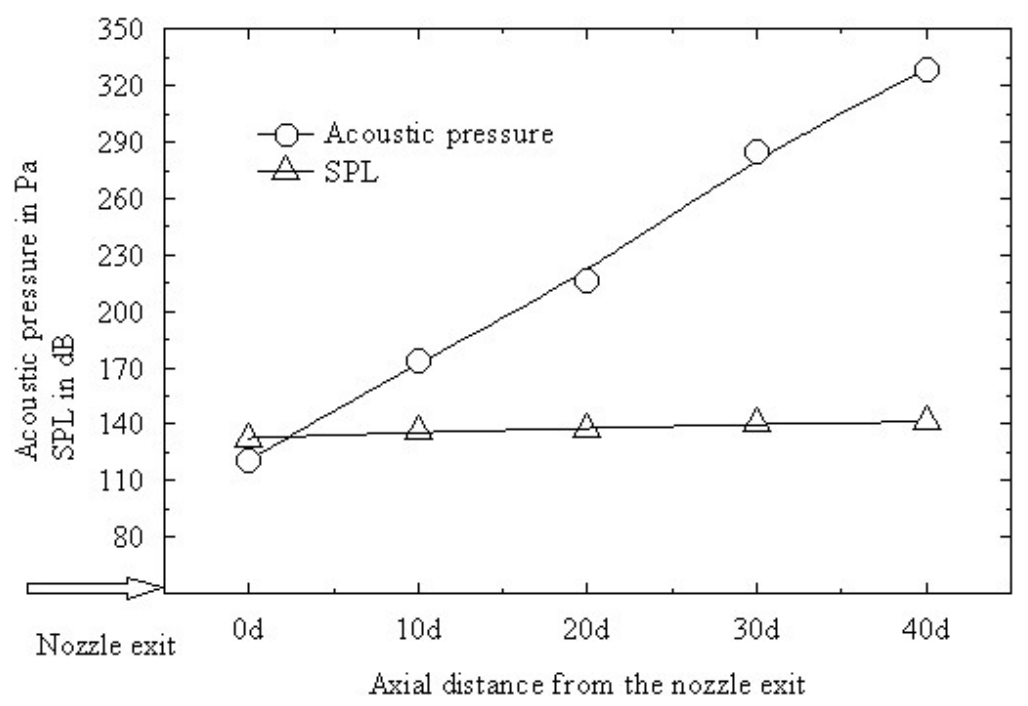

Figure 5. Variation of SPL and acoustic pressure at the jet boundary.

The surface source strength distribution at the jet boundary obtained by holography can be used in the wave equation solver to obtain the solution and hence predictive capability. Experimental values closely match the results obtained by acoustic holography. The percentage error for acoustic pressure is less than $11 \%$ and for SPL it is almost less than $3 \%$ overall.

The authors would like to thank the Vikram Sarabhai Space Centre, Thiruvananthapuram for providing the grant for carrying out the experimental work. We wish to express sincere thanks to M/s Manjunath Bhat, V Nagarajan and V Sriharsha for their help in conducting the experiments.

\section{List of symbols}

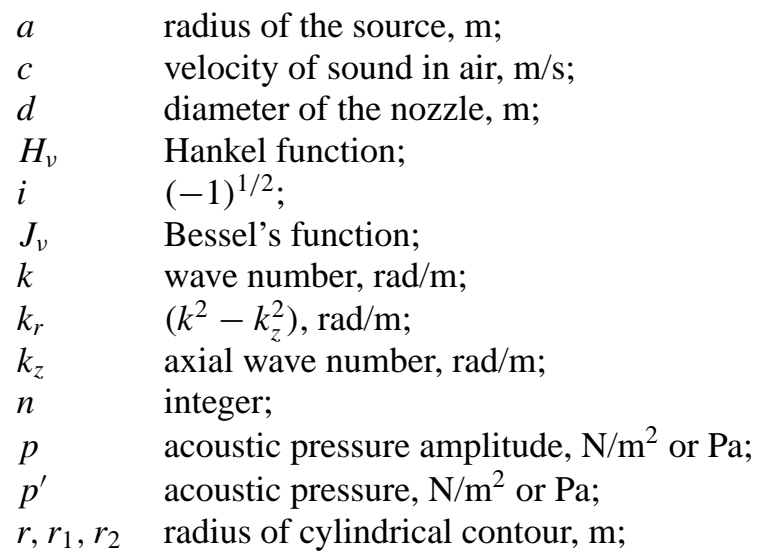


$\begin{array}{ll}t & \text { time, } \mathrm{s} ; \\ v & \text { acoustic velocity, } \mathrm{m} / \mathrm{s} ; \\ r, \theta, z & \text { polar coordinates; } \\ \lambda_{z} & \text { axial wave length, } \mathrm{m} ; \\ \omega & \text { angular frequency, } \mathrm{rad} / \mathrm{s} ; \\ \nabla & \frac{\partial}{\partial r^{2}}+\frac{1}{r} \frac{\partial}{\partial r}+\frac{\partial^{2}}{\partial \theta^{2}}+\frac{\partial^{2}}{\partial z^{2}} \text { (Laplacian operator). }\end{array}$

\section{References}

Coleman D D, Snedeker S R 1971 A study of free jet impingement. Part 1. Mean properties of free and impinging jets. J. Fluid Mech. 45: 281-319

Kreyszig E 1993 Advanced engineering mathematics (New York: John Wiley \& Sons)

Maynard J D, Williams E G, Lee Y 1985 Near-field acoustic holography - I. Theory of generalized holography and the development of NAH. J. Acoust. Soc. Am. 78: 395-1413

Morse P, Feshbach H 1953 Methods of theoretical physics (New York: McGraw Hill) pp 1265

Ribner H S 1958 On the strength distribution of noise sources along a jet. UTIA Report No. 51

Stephanishen P R, Benjamin K C 1982 Forward and backward projection of acoustic fields using FFT methods. J. Acoust. Soc. Am. 71: 803-812

Stone J R 1974 Interim prediction method for jet noise. NASA TMX-71618

Tennekes H, Lumley J L 1997 A first course in turbulence (Cambridge, MA: MIT Press).

Veronesi W A, Maynard J D 1987 Near-field acoustic holography - II. Holographic reconstruction algorithms and computer implementation. J. Acoust. Soc. Am. 81: 1307-1322

Williams E G, Maynard J D 1980 Holographic imaging without the wavelength resolution limit. Phys. Rev. Lett. 45: 554-557 